\title{
Les plaisirs cruels de la « plèbe » : combats de taureaux et représentation politique des bêtes dans la Grande-Bretagne de la première moitié du XIX siècle
}

The Cruel Pleasures of the "Plebeians": Bull-Baiting and the Political Representation of Animals in Early Nineteenth-Century Britain

Die grausamen Freuden des Pöbels. Bull-baiting und die politische Repräsentation der Tiere in Großbritannien in der ersten Hälfte des 19. Jahrhunderts

\section{Fabien Carrié}

\section{(2) OpenEdition}

\section{Journals}

Édition électronique

URL : http://journals.openedition.org/rh19/5186

DOI : $10.4000 /$ rh 19.5186

ISSN : $1777-5329$

\section{Éditeur}

La Société de 1848

\section{Édition imprimée}

Date de publication : 1 août 2017

Pagination : 63-79

ISSN : 1265-1354

\section{Référence électronique}

Fabien Carrié, «Les plaisirs cruels de la «plèbe » : combats de taureaux et représentation politique des bêtes dans la Grande-Bretagne de la première moitié du XIX siècle ", Revue d'histoire du XIXe siècle [En ligne], 54 | 2017, mis en ligne le 01 juillet 2019, consulté le 02 janvier 2020. URL : http:// journals.openedition.org/rh19/5186; DOI : 10.4000/rh19.5186 


\section{FABIEN CARRIÉ}

\section{Les plaisirs cruels de la "plèbe" : combats de taureaux et représentation politique des bêtes dans la Grande-Bretagne de la première moitié du $X I X^{e}$ siècle}

Au mois de mai 1809, l'avocat Thomas Erskine, célèbre pour ses nombreux engagements charitables et pour avoir défendu, contre ses propres convictions, des militants jacobins ${ }^{1}$, présente devant la chambre des Lords un projet de lois aux visées zoophiles ${ }^{2}$, qui entend interdire et punir les actes de cruauté commis à l'encontre des animaux domestiques ${ }^{3}$. La proposition de législation prévoit un large domaine d'application, le parlementaire souhaitant rendre illégales des pratiques aussi variées que les courses contre le temps ${ }^{4}$, les coups injustifiés portés contre les animaux de trait et de transport par les conducteurs d'attelages, l'affamement volontaire par les équarisseurs des chevaux de réforme en vue de précipiter leur trépas. De cet inventaire fourni, se trouve toutefois exclue une activité bien particulière: le bullbaiting, forme insulaire des combats d'animaux qui oppose des chiens à un taureau, cette omission devant prévenir toute objection à l'édiction du projet de loi ${ }^{5}$. Instruit des échecs de précédentes propositions législatives, Erskine cherche par là à désamorcer les critiques suscitées par la mise en cause d'un jeu dévolu aux seules classes populaires, les propositions antérieures ayant été perçues par de nombreux commentateurs comme autant de dispositions répressives de la plèbe et de ses pratiques. Par la mise à distance de cette question du bull-baiting, le parlementaire entend ainsi se présenter comme au-dessus des luttes entre les groupes et les partis, le dispositif répressif qu'il promeut devant s'appliquer à chacun, quelle que soit son origine sociale.

1. David Lemmings, 'Erskine, Thomas, first Baron Erskine (1750-1823)', Oxford Dictionary of National Biography, Oxford/New York, Oxford University Press, 2004.

2. Zoophile est entendu ici dans son sens premier, d'amour et de bonté envers les animaux.

3. Thomas Erskine, Cruelty to Animals. The speech of Lord Erskine in the House of Peers on 15th May 1809, on the Second Reading for Preventing Malicious and Wanton Cruelty to Animals, London, 1824.

4. Les courses contre le temps consistaient en des courses de chevaux sur de longues distances, le but du jeu étant de réaliser le trajet dans le temps le plus court. Il n'était pas rare que les chevaux, très durement sollicités, meurent d'épuisement à la suite de l'épreuve.

5. Ibidem. 
Mais hélas pour Erskine et ses soutiens, le projet de loi est défait à l'occasion de son passage devant la Chambre des communes. Malgré les précautions prises, la pratique du bull-baiting revient très vite au centre des débats, le potentiel discriminant vis-à-vis de la plèbe d'une législation zoophile ${ }^{6}$ se trouvant dès lors à nouveau questionné

La déconvenue d'Erskine est exemplaire de l'importance prise par cette forme bien particulière de jeu qu'est le combat de taureaux dans les luttes du moment autour de la définition des relations légitimes avec les bêtes. Le contexte est en effet particulièrement propice à ce genre de questionnements. La Grande-Bretagne est alors le théâtre tout à la fois de la création des premières organisations constitutives de la cause animale ${ }^{8}$, d'une inflation considérable des textes plaidant la bonté à l'égard des bêtes et de la multiplication des projets de lois zoophiles, à l'image de la proposition de 1809. Ces différents éléments constituent autant d'expressions du développement contemporain de l'idée d'une représentation sociale et politique des intérêts supposés des animaux. Ils sont les prémices de la constitution d'une nébuleuse idéologique "animaliste" déclinée au fil du temps en une série d'acceptions variées, prenant pour objet des pratiques différenciées et des formes spécifiques d'interaction avec les bêtes'. Dans les premières décennies $\mathrm{du}$ XIX ${ }^{\mathrm{e}}$ siècle au Royaume-Uni s'impose ainsi progressivement, sur fonds de controverses houleuses autour du jeu du bull-baiting, une définition de ce porte-parolat centré de manière privilégiée sur les pratiques et les attitudes des classes populaires vis-à-vis des animaux domestiques. Cette définition conditionne en retour le traitement de la question des relations avec les bêtes durant une majeure partie du XIX ${ }^{\mathrm{e}}$ siècle. On se propose ici de restituer le processus d'affirmation et de légitimation de cette déclinaison de l'idéologie "animaliste", à partir d'une analyse du jeu violent des combats de taureaux et des fortes réactions qu'il suscite dans la période en Grande-Bretagne. À l'encontre d'une "histoire sainte» qui considère cette prise de parole comme résultante d'un «choc moral» induit par le spectacle de la souffrance des bêtes, tout autant que d'une "histoire démonologique», décelant au contraire dans l'engagement pour les sans-voix à quatre pattes les calculs de la classe

6. Par législation zoophile, on entend l'ensemble des textes de lois et règlements visant à prohiber, dans un souci de respect de la morale et de maintien de l'ordre public, les violences à l'encontre des animaux.

7. Cobbet's Parliamentary Debates, London, R. Bagshaw, 1804-1812, volume 14, p. 553-571, 804808, 851-853, 889-890, 1029-1032. Cf. Emma Griffin, Blood Sport: Hunting in Britain since 1066, New Haven/London, Yale University Press, 2007. 2011.

8. Christophe Traïni, La cause animale (1820-1980). Essai de sociologie historique, Paris, PUF,

9. À ce sujet, je me permets de renvoyer à Fabien Carrié, Parler et agir au nom des "bêtes": production, diffusion et réception de la nébuleuse idéologique "animaliste" (France et Grande-Bretagne, 1760-2010), thèse de science politique sous la direction de Bernard Pudal, Nanterre, Université Paris Ouest Nanterre, 2015. Ce travail s'inscrit dans le sillage d'une histoire sociale des idées politiques et s'efforce de restituer la carrière et la trajectoire de cette idée de représentation sociale et politique des animaux, ses déplacements et réappropriations successives par différents groupes sociaux. 
dominante pour renforcer son autorité, il s'agira de montrer l'intérêt, pour rendre compte du phénomène, d'une analyse pluricausale attentive aux jeux d'échelle entre différents niveaux d'intégration sociale. Le développement dans la période d'une entreprise de représentation des animaux victimes des violences des classes populaires s'apprécie ainsi dans l'articulation des dynamiques internes qui affectent la configuration même du jeu et des redéfinitions des rapports de force au niveau du champ du pouvoir et de l'espace public britannique. Afin de mener à bien cette étude, ont été mobilisés la presse généraliste et sportive du moment, tout un ensemble d'encyclopédies, de manuels et de dictionnaires traitant des loisirs populaires, de même encore que de nombreux récits de vie, autobiographies et recueils de correspondance dans lesquels la pratique incriminée du bull-baiting se trouve évoquée. À cela s'ajoute la recension systématique des débats parlementaires de la période ayant portés sur les projets de législation zoophile et, plus largement, l'examen des prises de position dans l'espace public des représentants de l'élite sociale britannique sur ce sujet, prises de position à chaque fois rapportées aux propriétés sociales et aux positions occupées par ces agents.

\section{LES COMBATS DE TAUREAUX À L'ORÉE DU XIX SIÈCle : UNE CONFIGURATION FRAGILISÉE}

La restitution des logiques et des mécanismes qui régentent la pratique du bull-baiting peut aider, à un premier niveau d'analyse, à mieux saisir les raisons de la focalisation des critiques sur ce jeu décrié. Encore faut-il prendre garde aux réflexes culturalistes et ethnocentriques que ne manquera pas de susciter la description de l'activité chez le lecteur contemporain, rarement accoutumé à la souffrance et à la mort, y compris animales. Certes, le sport se révèle brutal et sanglant, tant dans son déroulement que dans sa finalité : un taureau attaché à l'aide d'une longe à un piquet solidement ancré dans le sol est confronté dans un combat à mort à des chiens - les fameux bull-dogs qui, au moment de la controverse, ne constituent pas encore une « race» aux traits bien définis ${ }^{10}$. Le jeu est scandé et rythmé par les phases d'attaque des chiens et se termine avec la mort du taureau, à la suite le plus souvent de son immobilisation, lorsqu'un bull-dog plus habile et rapide que les autres parvient à saisir et à maintenir dans l'étau de ses mâchoires les naseaux particulièrement sensibles du ruminant ${ }^{11}$. Mais si la pratique est violente, entrainant réguliè-

10. Harriet Ritvo, 'Pride and Pedigree: the Evolution of the Victorian Dog Fancy', Victorian Studies, $\mathrm{n}^{\circ}$ 29, 1986, p. 227-253; Grace Burchell, Breeding Frankenstein's Bulldog: Reimagining the Pedigree in Nineteenth Century England, Dissertation presented for the Honours Degree of BA, University of Nottingham, School of geography, 2015.

11. Cf. la description proposée par Delabere Pritchett Blaine, An Enclyclopaedia of Rural Sports: or a Complete Account (Historical, Practical, and Descriptive) of Hunting, Shooting, Fishing, Racing, etc. etc., London, Longmans, Green, Reader and Dyer, 1870, p. 129-130. 
rement des blessures mortelles chez la plupart des animaux combattants, elle ne constitue en rien une exception culturelle dans la Grande-Bretagne de la croisée des XVIII e et XIX ${ }^{e}$ siècles. Combats de coqs et de chiens, pratiques variées de vénerie et de braconne, blessures et coups quotidiennement et publiquement infligés aux bêtes de trait et de somme, mises à mort du bétail et découpage des viandes dans les étals de boucherie ouverts sur la rue : les interactions violentes avec les animaux sont omniprésentes dans la période, et sont encore exacerbées par l'inflation conjoncturelle sans précédent du nombre et des usages des bêtes ${ }^{12}$. L'espace social est ainsi saturé du spectacle de scènes et de formes de relations brutales vis-à-vis des animaux. Pourtant, quand bien même ces différentes pratiques suscitent les protestations croissantes d'une minorité de clercs et de littérateurs, l'indignation qu'elles soulèvent dans l'espace public britannique s'avère autrement moins importante et systématique que dans le cas du bull-baiting. Comprendre cette singularité suppose dès lors de pousser plus loin l'analyse de ce jeu, en l'envisageant dans la droite ligne des travaux de Norbert Elias et de Eric Dunning comme une configuration impliquant à la fois des humains et des animaux, dont il s'agit de restituer les dynamiques et les tensions, l'historicité et les inflexions successives ${ }^{13}$.

Le bull-baiting à l'aube du XIX ${ }^{\mathrm{e}}$ siècle désigne une activité ancienne, pluriséculaire, dont les origines largement mythifiées remonteraient au XIII ${ }^{e}$ siècle. Elle est le fruit du croisement de la pratique quotidienne d'abattage des bouchers, souvent aidés dans leurs tâches d'auxiliaires canins, des loisirs martiaux de la noblesse et des jeux sanglants importés sur l'île britannique pendant l'antiquité suite à l'invasion romaine ${ }^{14}$. Implanté dans de nombreux villages et villes de Grande-Bretagne, où il se décline dans des variantes locales, le jeu jouit pendant longtemps d'une popularité qui transcende les classes, les ordres et les partis ${ }^{15}$. Cette popularité se traduit matériellement dans le nom des rues de nombreuses localités : on trouve ainsi fréquemment des voies baptisées "Bull Stake» ou «High Bullen» dans les villes et villages britanniques jusqu'au XVIII ${ }^{e}$ siècle $^{16}$. De même en est-il de la présence récurrente au sein de ces territoires de bull-rings, petites arènes sises le plus souvent sur les places du marché ${ }^{17}$. L'engouement pour la pratique

12. Jason C. Hribal, 'Animals, Agency, and Class: Writing the History of Animals from Below', Human Ecology Review, tome 14, n 1, 2007, p. 101-112.

13. Eric Dunning and Norbert Elias, The Quest for Excitement: Sport and Leisure in the Civilising Process, Dublin, University College Dublin Press, 2008 (1993).

14. 'Bull-Baiting', The Sporting Review. A Monthly Chronicle, janvier 1851, p. 454 ; Jacques Marsan de Perramont, Les révolutionnaires tels quils sont et la vérité vengée : ouvrage moral et politique, Bordeaux, Lavigne Jeune, 1817.

15. Douglas A. Reid, 'Beasts and Brutes: Popular Blood sports c. 1780-1860', in Richard Holt (ed.), Sport and the Working Class in Modern Britain, Manchester/New York, Manchester University Press, 1990, p. 12-28; Frederick W. Hackwood, Old English Sports, London, T. T. Unwin, 1907.

16. John Garland, 'Bull-baiting', Notes and Queries, $2^{\text {nd }}$ series, $n^{\circ} 101,5$ December 1857, p. 460 ; 'Bull-baiting in England', ibidem, Gth serie, n' 1, 27 mars 1880, p. 265.

17. 'Bull Rings', Notes and Queries, 13 juin 1942, p. 331. 
s'objective jusque dans les codes et règlements locaux, qui prohibent la vente de viande de taureau à moins que l'animal n'ait été préalablement tué dans le cadre d'une session de bull-baiting ${ }^{18}$.

La pratique n'a manifestement guère évolué au fil du temps dans son déroulement et ses modalités. Une fois le taureau acquis, le plus souvent offert par tel ou tel membre de la notabilité locale ${ }^{19}$, l'annonce du combat est diffusée, incitant les propriétaires de chiens des alentours à participer au jeu en l'échange d'une rétribution matérielle (repas gratuit et/ou argent) ${ }^{20}$. Ces conditions de possibilité remplies, le jour du combat se met en place une configuration singulière, où se mêlent et interagissent dans une confusion certaine un taureau et un public, des chiens et leurs propriétaires. Les quelques rares récits existants de la dynamique de jeu donnent à voir en effet une activité très faiblement régulée, caractérisée par l'absence de toute instance d'arbitrage et de contrôle, par l'étanchéité toute relative des partitions entre le public humain et les belligérants à quatre pattes. À l'image des spectateurs du théâtre shakespearien de la période moderne qui n’hésitaient pas à intervenir sur scène et à agir sur le contenu et le déroulement de la pièce ${ }^{21}$, l'assistance réunie à l'occasion des bull-baitings est loin d'être passive et intervient bien souvent directement dans l'arène. Un témoin relate ainsi avoir vu lors d'un combat un spectateur se joindre à la mêlée et venir mordre le taureau de concert avec la meute ${ }^{22}$. Il est par ailleurs courant d'assister à l'occasion des assauts des bull-dogs à la course périlleuse du possesseur d'un chien, tentant d'attraper au vol son champion, encorné et propulsé dans les airs par le taureau, tout en s'efforçant d'esquiver les ruades et les coups de cornes ${ }^{23}$. Ces exemples témoignent tous d'une même tendance, d'un brouillage des limites des catégories d'animalité et d'humanité, d'un relâchement général au sein de ces configurations des mécanismes de contrôle des violences pulsionnelles. Envisagée dans cette perspective, la configuration détonne et dénote un important décalage avec une majorité des formes contemporaines de sports impliquant des animaux. C'est le cas par exemple de la chasse à courre, des combats de chiens et de coqs, tous codifiés et dotés d'une régulation, ne serait-ce qu'a minima, qui instaure progressivement une frontière et des partitions nettes entre l'activité agonistique réservée aux bêtes et les

18. 'Bull-baiting', Notes and Queries, 9th Series, $\mathrm{n}^{\circ}$ 9, 8 mars 1902, p. 188; 'Baiting of Slaughter Cattle', ibidem, 27 octobre 1928, p. 302; C. A. Luttrel, 'Baiting of Bulls and Boars in the Middle English "Cleanness', ibidem, 19 janvier 1952, p. 23-24. Le goût très fort et marqué de la viande de taureau est réputé s'adoucir sous l'effet de l'excitation du combat, le bull-baiting étant dès lors considéré comme un procédé nécessaire pour rendre la chair de l'animal propre à la consommation.

19. The Times, $\mathrm{n}^{\circ} 10745,8$ October 1819 , p. 2.

20. Notes and Queries, 5th series, $\mathrm{n}^{\circ} 22,25$ October 1879, p. 328 ; 'Bull-baiting in England', ibidem, 6th Series, ${ }^{\circ} 1,27$ March 1880, p. 265.

21. Lawrence W. Levine, Highbrow-Lowbrow: The Emergence of Cultural Hierarchy in America, Cambridge (Mass.), Harvard University Press, 1988, traduction française Culture d'en haut, culture d'en bas. L'émergence des hiérarchies culturelles aux États-Unis, Paris, La Découverte, 2010.

22. 'Bull-baiting: Bull-rings', Notes and Queries, $2^{\text {nd }}$ series, $n^{\circ} 106,9$ January 1858, p. 35.

23. G. Watkins Grubb, 'Cullompton in Devonshire; the Parish and the Parson', Notes and Queries, 5 October 1946, p. 141-143. 
postures plus distanciées des participants humains à ces jeux ${ }^{24}$. La porosité et le flou des frontières, le maintien durable d'un seuil très élevé de tolérance vis-à-vis de la brutalité et du sang au sein de cette configuration expliquent sans doute pour une large part la force du stigmate apposé au bull-baiting à l'orée du XIX ${ }^{\mathrm{e}}$ siècle.

Il faut bien sûr se garder de tout réalisme de premier degré dans la lecture des témoignages disponibles sur la pratique. Comme pour de nombreuses activités propres aux milieux populaires, on ne dispose ici que de trop rares sources de première main. Dans les matériaux mobilisables au sujet du bullbaiting, les descriptions de chroniqueurs sportifs et de journalistes le plus souvent critiques d'un jeu considéré comme barbare le disputent à quelques mises en récit tardives de souvenirs d'enfance, probablement altérés par les représentations négatives et les connotations péjoratives associées au jeu dans la période récente. Si l'insistance dans les écrits disponibles sur la bestialité du loisir se conçoit dès lors pour une large part comme relevant d'une logique de disqualification, la description des caractéristiques violentes prêtées au bull-baiting n'en est pas pour autant sujette à caution. La brutalité du loisir, comme la participation active des spectateurs humains aux combats, s'avèrent des conditions bien souvent nécessaires au maintien dans la configuration d'une tension suffisante entre les protagonistes canins et bovins pour profiter d'un "bon jeu» ("good game»). Dans une période où s'impose une définition de la pratique sportive comme un savant et délicat équilibre entre la tension de la poursuite et l'excitation causée par le combat et la victoire sur l'adversaire $^{25}$, les participants humains au bull-baiting mobilisent et mettent à profit les moyens limités à leurs dispositions pour s'assurer de la réussite de l'activité. L'usage de la violence par les spectateurs à l'intérieur de l'arène constitue dès lors un mode privilégié de régulation du sport et de maintien de l'équilibre des forces entre le taureau et les chiens. Ainsi peut-on comprendre l'usage régulièrement mentionné de piques ou d'épieux, enfoncés dans les flancs des bull-dogs parvenus trop rapidement à mordre les naseaux de l'adversaire, afin de leur faire lâcher prise. On peut supposer que la même logique gouverne cet acte rapporté (avec désapprobation) du maître d'un chien trop vivace et féroce, estropiant son animal pour faire durer un jeu au départ inégal et faire monter les paris; de même encore que ces coutures et sutures réalisées à la hâte sur un champion canin éventré par un taureau, afin qu'il poursuive un assaut trop vite arrêté ${ }^{26}$. Les chiens ne sont pas les seules victimes de ces interventions et de ces formes brutales d'équilibrage de la dynamique de jeu : il n'est pas rare en effet que les taureaux peu disposés à combattre soient sti-

24. La comparaison du jeu du bull-baiting avec les fameux combats de coqs balinais décrits par Clifford Geertz, forme exemplaire de combats d'animaux très lourdement codifiés, est ici particulièrement éclairante. Cf. Clifford Geertz, "Jeux d'enfer. Notes sur le combat de coqs balinais", Le Débat, tome 7, n 7, 1980, p. 86-146.

25. Eric Dunning and Norbert Elias, The Quest for Excitement..., op. cit

26. The Christian Observer, volume 1, 1802, p. 433. 
mulés et incités par les spectateurs humains, qui souvent leurs brisent les os de la queue afin de les exciter, ou placent des brandons enflammés sous leurs corps pour les forcer à se dresser sur leurs pattes et à lutter ${ }^{27}$. À défaut d'autres ressources à disposition, l'usage de la force dans les interactions de face à face entre humains et animaux au sein de ces configurations permet de maintenir un rythme satisfaisant durant l'intégralité du jeu et explique pour une large part le chaos apparent et le niveau élevé de brutalité du sport. Le contraste avec la chasse aux renards, loisir privilégié de la gentry et de l'aristocratie britannique dont les principes et les règles se stabilisent au cours des XVII ${ }^{e}$ et XVIII ${ }^{\mathrm{e}}$ siècles, est à ce titre saisissan ${ }^{28}$. L'accumulation préalable de ressources, tout à la fois matérielles et symboliques, permet aux agents humains de ces configurations d'adopter des postures surplombantes, de maintenir une forme de distance vis-à-vis de la course-poursuite entamée entre le renard et la meute des chiens. Les ressources mises en œuvre sont multiples : usage d'auxiliaires aux rôles et aux fonctions variés (meute de chiens de chasse, chevaux, mais aussi rabatteurs humains), manipulations et sélections génétiques opérées sur des variétés de chiens au flair et à l'endurance dorénavant très développés les rendant particulièrement aptes à la traque, traditions d'élevage canin et d'équitation transmises au sein des familles de l'élite patricienne britannique. Elles dispensent les chasseurs à courre de toute intervention directe sur le déroulé de la poursuite et les placent dans des positions d'observateurs passifs d'une chasse et d'une mise à mort dont le caractère violent se trouve de fait comme atténuéer .

Conditions de l'euphémisation de la violence, l'accumulation et la concentration de ressources comme celles que peuvent mobiliser les adeptes aristocrates de la cynégétique s'avèrent impossibles dans le cas du bull-baiting, devenu au fil des siècles une pratique exclusive de la plèbe. Bien qu'il ne soit qu'un rituel violent parmi tant d'autres ${ }^{30}$, le jeu se voit ainsi progressivement exposé du fait de l'incapacité de ses soutiens à l'adapter et à l'ajuster matériellement et symboliquement aux normes évolutives de tolérance à la brutalité et au sang. Cette situation est encore renforcée à la croisée des XVIII et $\mathrm{XIX}^{\mathrm{e}}$ siècles par une évolution conjoncturelle, qui grève encore un peu plus les chances de pérennisation de la pratique. Des travaux récents ont en effet mis au jour un phénomène de reflux du soutien, financier pour l'essentiel, apporté jusque-là par l'élite sociale et la bourgeoisie marchande britannique à l'organisation et à la bonne tenue du bull-baiting ${ }^{31}$. Ce désengagement initié

27. 'Bull-baiting', The Times, $\mathrm{n}^{\circ} 11684,8$ October 1822, p. 3.

28. Allyson Nancy May, The Fox-hunting Controversy, 1781-2004, Farnham, Ashgate, 2013.

29. Idem.

30. Ainsi par exemple des exécutions publiques, Valentine A. C. Gatrell, The Hanging Tree: Execution and the English People, 1770-1868, Oxford, Oxford University Press, 1994; cf. plus largement Charles Tilly, The Politics of Collective Violence, Cambridge, Cambridge University Press, 2003.

31. Emma Griffin, 'Sports and Celebrations in English Market Towns, 1660-1750', Historical Research, tome $75, \mathrm{n}^{\circ} 188$, may 2002, p. 188-208. 
au cours du XVII e siècle au moment de la Glorieuse Révolution, n’a fait que s'accentuer depuis lors, et ce même après la restauration monarchique. Si l'on peut déceler dans les changements de comportement de l'élite et de la middle class locales l'expression d'un malaise accru face à un sport à la brutalité par trop voyante et prononcée, c'est sans doute tout autant l'affirmation de la spécialisation des places de marché, vouées désormais à la seule conduite des activités économiques, qui se joue dans l'arrêt des financements marchands de l'entretien des bull-rings ${ }^{32}$. Quelle que soit la part de ces différents facteurs dans la disparition progressive de ce secours matériel, ses effets sur la conduite du loisir s'avèrent bien vite considérables.

L'opposition de la bourgeoisie marchande et commerçante à l'organisation de sessions du jeu dans des espaces publics aux dimensions par ailleurs de plus en plus réduites du fait de l'amplification du mouvement des enclosures $^{33}$, l'arrêt du financement de l'entretien des bull-rings et leur dégradation consécutive, contraignent finalement les amateurs à organiser les combats dans d'autres lieux, bien souvent inadaptés à la pratique du sport ${ }^{34}$. Dès lors, les incidents se multiplient, parfois mortels. C'est le cas du plus célèbre d'entre eux, largement relayé par la presse locale et nationale de l'époque, qui eut lieu dans la ville de Rochdale, dans le nord-ouest de l'Angleterre : à l'occasion d'un match installé aux abords d'une rivière, un pont de pierre sur lequel s'était massé le public s'effondra partiellement du fait de la masse trop importante des spectateurs, entrainant la mort de plusieurs d'entre eux ${ }^{35}$. Autre conséquence, aux implications plus rarement dramatiques, l'échappée sauvage des taureaux parvenant à rompre leurs longes ou à déterrer du sol le piquet auquel ils étaient attachés semble être devenue à partir du XVIII ${ }^{e}$ siècle un aléa fréquent du sport, qui suscite chez les commentateurs et les témoins une réprobation de plus en plus unanime ${ }^{36}$. En définitive, la violence de la pratique, jusque-là plus ou moins confinée dans les limites des bull-rings régulièrement entretenus, se trouve comme "dé-compartimentée ", du fait du tarissement de la manne financière des notabilités locales. Cette soudaine mise au jour contribue à rendre intolérable et à publiciser la brutalité du bull-baiting ${ }^{37}$.

32. Idem; cf. par ailleurs H. Prosser Chanter, 'The Parish Bull', Notes and Queries, 27 december 1924 , p. 469.

33. Jeannette M. Neeson, «La clôture des terres et la société rurale britannique : une revue critique", Histoire, économie et société, tome 18, nº 1, janvier-mars 1999, p. 83-106; Gérard Béaur [dir.], La Terre et les hommes. France et GB, XVII'-XVIII siècles, Paris, Hachette-Pluriel, 1998.

34. 'The King v. William Butler, Martin Martin and William Williams', The Times, $\mathrm{n}^{\circ} 11$ 008, 10 August 1820, p. 3.

35. 'Dreadful Accident', The Times, $\mathrm{n}^{\circ} 11$ 089, 13 November 1820, p. 3.

36. 'Bull-baiting in England', Notes and Queries, 5th serie, $n^{\circ} 22,6$ December 1879, p. 455-456; The Christian Observer, volume 1, 1802, p. 432-433; 'The Bury Paper of Tuesday gives the Following Account of a Bull-bait', The Times, $n^{\circ} 5$ 271, 24 November 1802, p. 3.

37. Sur les logiques de compartimentation de la violence, $c f$. Abram de Swaan, The Killing Compartments: the Mentality of Mass Murder, New Haven (Conn.), Yale University Press, 2015, traduction française Diviser pour tuer : les régimes génocidaires et leurs hommes de mains, Paris, Seuil, 2016. 
Un article du Times daté du 22 juin 1787, relatant la tenue dans les alentours des jardins royaux de Kensington d'un combat alors que s'y déroulait parallèlement une réception princière, le donne nettement à voir. Insistant sur les fuites successives du taureau parvenu à rompre ses liens, l'auteur y fustige la violence grossière et la dangerosité du jeu de la plèbe, encore accentuées par la proximité immédiate du faste et des plaisirs raffinés des "grands»: «Tandis que la noblesse et la gentry s'amusaient entre elles dans les Jardins Royaux de Kensington, la plèbe entreprenait de se divertir du côté nord des Jardins, face à la nouvelle entrée, en faisant combattre un jeune taureau. [...] Heureusement pour les spectateurs, le taureau avait plus d'humanité et de courtoisie que ceux assemblés pour se distraire de ses souffrances : la corde se détacha du piquet, et le taureau, désireux d'échapper à ses oppresseurs, se tourna vers la route. Les spectateurs effrayés prirent la fuite et, dans la panique, nombre d'entre eux furent jetés à terre. Le taureau arriva à l'endroit où les fils de la cruauté et de la folie étaient étalés sur le sol, mais le noble et généreux animal dédaigna de prendre l'avantage, ou de leur infliger une punition exemplaire ${ }^{38}$. Avec cette dynamique croissante de "dé-compartimentation", tout se passe comme si la violence intrinsèque au bull-baiting, ses dysfonctionnements et les actes de résistance des bêtes prises dans la configuration devenaient soudainement visibles et signifiants pour de nombreux agents sociaux extérieurs à la pratique. Dans un contexte marqué par le développement de l'idée d'une représentation politique des animaux victimes de cruautés, le combat des bull-dogs et des taureaux va dès lors se trouver placé au premier plan des débats qui agitent l'espace public.

\section{LA POLITISATION DE LA VIOLENCE DU BULL-BAITING DANS LE CHAMP DU POUVOIR}

La brutalité et la violence du bull-baiting, révélées et comme amplifiées du fait des dynamiques qui affectent l'équilibre et les mécanismes de la configuration du jeu, vont trouver un écho particulier dans les textes et les discours des premiers promoteurs de la cause animale, produits à partir de la fin du XVIII siècle. Dans les plaidoyers des tenants de cette idéologie "animaliste» pour une redéfinition des traitements et des modalités d'interaction avec les bêtes, la critique de la pratique «barbare» des combats opposant chiens et taureaux trouve rapidement sa place. Mais si la contestation du bull-baiting prend une bonne part dans les prises de position de ces porte-parole, elle n'exclut pas la mise en cause d'autres activités impliquant des animaux. Aux côtés de ce jeu, se trouvent également questionnées des pratiques socialement plus transversales comme les combats de coqs, de même encore que les différentes déclinaisons de la chasse à courre et de la cynégétique, alors apanages

38. The Times, $\mathrm{n}^{\circ} 780,22$ June 1787, p. 2. 
incontestés de l'aristocratie et de la gentry. Produite par un groupe hétérogène de publicistes "gardiens de la morale", venant du clergée $e^{39}$, et de littérateurs et de littératrices spécialisés dans la publication d'ouvrages pour enfants ${ }^{40}$, l'idée "animaliste" s'adresse en effet d'abord à un public issu d'une middle-class britannique imprégnée de valeurs puritaines, volontiers hostile aux loisirs profanes et soucieuse de se distinguer dans ses pratiques de la plèbe comme de l'élite patricienne. Comme l'explique l'un des principaux contributeurs à l'idée contemporaine d'un porte-parolat moralisant des animaux, le pasteur anglican Humphry Primatt, c'est bien la société dans son ensemble qu'il faut purger de ses inclinations cruelles, le bull-baiting n'étant finalement qu'une émanation parmi d'autres d'une tendance générale :

«L'indulgence fautive des parents, les instances variées de cruauté sportive, sous une forme ou une autre, pratiquées quotidiennement, à tous les niveaux de la société, par des personnes dont la conduite à d'autres égards peut être vraiment charmante et respectable, de même encore que les nombreuses pratiques barbares favorisées et parfois même approuvées par des hommes de pouvoir ou de haut rang, nous conduisent à considérer les animaux comme des créatures insensibles et insignifiantes, faites seulement pour notre plaisir et pour le sport. Et lorsque nous réfléchissons aux barbaries choquantes, et que nous voyons la rage brutale exercée par les hommes les plus vils, sans contrôle de la loi et sans remarque ou reproche adressés depuis l'autel, nous sommes presque tentés de tirer cette conclusion, que la cruauté ne peut pas être un péché » ${ }^{41}$.

Pour comprendre la politisation de ce rituel violent et la focalisation croissante dans les premières décennies du XIX ${ }^{\mathrm{e}}$ siècle des promoteurs d'un porte-parolat des bêtes sur les pratiques des classes populaires, il faut dès lors s'intéresser à d'autres niveaux d'intégration sociale que la seule configuration de ce jeu controversé. C'est en effet à l'occasion de la mise en circulation de l'idée «animaliste» en direction du champ du pouvoir, autour de 1800, que s'opère ce resserrement des critiques et des luttes autour du bull-baiting. La période est marquée par le développement de tensions concernant le domaine juridique et des problématiques de maintien de l'ordre. Aux partisans de la réaffirmation du système ancestral de domination par la mise en scène d'une violence accrue contre la plèbe, s'opposent les tenants d'un renforcement des forces de police et de l'autorité judiciaire, qui peinent alors à légitimer un modèle d'organisation perçu comme inadapté à la société bri-

39. Gisèle Sapiro, "Modèle d'intervention politique des intellectuels. Le cas français ", Actes de la recherche en sciences sociales, 2009/1, $\mathrm{n}^{\circ} 176-177$, p. 8-31.

40. Tess Cosslett, Talking Animals in British Children's Fiction, 1786-1914, Aldershot, Ashgate, 2006.

41. Humphry Primatt, Duty of Mercy and Sin of Cruelty to Beasts and Sin of Cruelty to Brute Animals, London, R. Hett, 1776, p. 14-15. 
tannique ${ }^{42}$. C'est dans ce contexte que s'impose la question du traitement des bêtes dans l'espace public, avec l'émergence d'un mouvement de réforme morale porté par la bourgeoisie marchande. Sur fond de crise des rapports traditionnels d'autorité, des militants de groupes comme la Society for the suppression of vice entendent remettre de l'ordre en imposant à tous l'obéissance à des normes éthiques renouvelées, telles que le refus de la cruauté vis-à-vis des animaux ${ }^{43}$. Le lobbying et l'activisme de ces collectifs vont notamment permettre le dépôt et la discussion au sein du parlement des premiers projets de lois visant à pénaliser certains actes labellisés comme cruels ${ }^{44}$. Dans les débats qui s'ensuivent, le bull-baiting en vient très vite à occuper une place centrale, éclipsant toute une série d'autres pratiques comme la vénerie ou les vivisections, pourtant considérées comme également problématiques par les pionniers lettrés de la cause animale. Les trois premières propositions présentées devant les chambres législatives en 1800, 1803 et 1805, bien que rédigées par des parlementaires différents, portent ainsi toutes sur ce seul sport et requièrent d'une même voix son abolition ${ }^{45}$. Quant aux projets de lois de $1809,1810,1821$ et 1824 , si leurs visées sont plus larges et ne concernent pas directement ou pas exclusivement le bull-baiting, force est toutefois de constater l'omniprésence du sport dans les échanges souvent vifs qui ponctuent l'examen des textes.

L'insistance sur le caractère violent de ce jeu, sur les multiples débordements qu'il entraine - depuis l'organisation de paris jusqu'aux accidents mortels publicisés par la presse, en passant par la consommation excessive d'alcool des spectateurs -, favorise la fixation des débats sur les pratiques des classes populaires et leurs interactions avec les animaux domestiques. Pour de nombreux parlementaires, soutiens comme adversaires des projets de législation zoophile, le bull-baiting va être progressivement perçu comme une manifestation exemplaire de la cruauté de la "plèbe», ceci justifiant la redéfinition partielle de l'idéologie "animaliste» dans le sens d'une stricte focalisation sur les activités des pauvres. Contre toute attente, cette recomposition de l'idée de porte-parolat des bêtes, opérée au sein du champ du pouvoir britannique à partir du cas du bull-baiting, ne se résume pas à une stratégie cynique et consciente des grands du royaume en vue de la défense de leurs intérêts et d'un contrôle accru des dominés. Elle s'apprécie plutôt comme

42. Cf. V. A. C. Gatrell, The hanging tree... op. cit.; Arthur Burns and Joanna Innes (eds), Rethinking the Age of Reform: 1780-1850, Cambridge/New York, Cambridge University Press, 2003.

43. M. J. D. Roberts, Making English Morals. Voluntary Association and Moral Reform in England, 1787-1886, Cambridge, Cambridge University Press, 2004; David Eastwood, 'Men, Morals and the Machinery of Social Legislation, 1790-1840', Parliamentary History, 13, 1994, p. 190-205.

44. Des projets de lois de cet ordre sont discutés respectivement en 1800, 1802, 1805, 1809, $1810,1821,1822$.

45. Hansard, The Parliamentary History of England, from the earliest period to the year 1803, volume XXXV, comprising the period from the the twenty-first day of March, 1800, to the twentyninth day of October 1801, p. 202-213; idem, volume XXXVI, p. 829-854. 
la rencontre entre plusieurs dynamiques concomitantes, à commencer par le processus même de transfert de l'idée en direction du champ du pouvoir.

Au sein du parlement, la novation symbolique de la prise de parole au nom des bêtes est introduite et saisie à l'orée du XIX ${ }^{e}$ siècle par un groupe restreint d'individus aux propriétés bien spécifiques. Militants aguerris des campagnes abolitionnistes ou défenseurs de la liberté de la presse, zélateurs ou sympathisants évangélistes proches, ou même intégrés aux réseaux de la Clapham Sect, les soutiens de l'entreprise de représentation des animaux donnent à voir à l'analyse une série de traits communs. Ils sont issus pour une part significative de familles récemment anoblies de la bourgeoisie marchande et financière et des classes capacitaires. À ceux-ci s'ajoutent quelques fils d'une noblesse désargentée, contraints par le sort et la mauvaise fortune à embrasser une carrière professionnelle, dans le domaine juridique notamment. Tous expérimentent des situations proches d'entre-deux, au principe de l'intériorisation tout à la fois d'un ethos aristocratique et des valeurs et dispositions éthiques partagées par les différentes fractions de la middle class. Les spécificités de leurs trajectoires et de leur habitus clivé expliquent pour partie les positions de passeurs qu'ils revendiquent, courroies de transmission au sein du parlement des principes moraux défendus par l'avant-garde de la bourgeoisie britannique. Il faut dire que leurs dispositions à l'engagement désintéressé trouvent facilement à s'actualiser au sein d'un champ du pouvoir alors structuré par les enjeux des luttes patrimoniales opposant les grandes et vieilles familles de l'aristocratie ${ }^{46}$. Pour exister et être audible au sein d'un tel cénacle, ces dominés des dominants vont se penser comme les représentants des grandes causes à visée moralisante qui se développent au sein de l'espace public, leur engagement en faveur du porte-parolat des bêtes s'inscrivant ainsi dans la continuité de leurs précédents investissements ${ }^{47}$.

Principaux soutiens des tentatives de législation zoophile, ces parlementaires vont grandement contribuer à infléchir le contenu et les contours de l'idée au sein des chambres. Ils défendent notamment la dimension éthique de la prise de parole au nom des bêtes, et ce y compris contre les initiateurs de certains des premiers projets de lois, qui voyaient dans la répression souhaitée des brutalités de la plèbe la potentialité d'une mesure préventive des révoltes populaires ${ }^{48}$. Les jeux contestés des pauvres doivent être condamnés pour leur barbarie intrinsèque et non pas en fonction des visées utilitaristes de domestication de la force de travail. S'ils ne négligent pas les profits d'ordre promis par une telle législation, ils insistent d'abord sur une conception dialectique de l'entreprise de représentation, dans laquelle les enjeux de contrôle social s'articulent au projet d'intéressement de la plèbe. Il s'agit par

46. Gerald E. Aylmer, 'The Peculiarities of the English State', The Journal of Historical Sociology, tome 3, no 2, June 1990 .

47. Thomas Erskine, op. cit.

48. $C f$. à ce sujet la lettre de William Wilberforce à Hannah More, publiée dans Robert I. and Samuel Wilberforce, The Life of William Wilberforce, London, 1838, volume 2, p. 36. 
la mise en œuvre du porte-parolat de favoriser l'intégration des dominés, de les façonner moralement en influant sur leurs pratiques et sur la qualité de leurs interactions avec les animaux. On comprend mieux dans cette perspective l'importance prise par le jeu du bull-baiting dans leur rhétorique. Tout en se gardant d'être assimilés à des ennemis des loisirs du peuple, ils fustigent une activité dont la violence jamais contenue aux combats entre animaux démontre à elle seule le caractère contagieux de la cruauté et de la barbarie, qui avilissent et souillent moralement l'ensemble des participants. Mettre un terme à ce sport brutal reviendrait à faire ouvre de salut public, en restaurant la dignité et les bonnes dispositions des membres des classes populaires. Filant la métaphore du jeu taurin, l'abolitionniste William Wilberforce fait ainsi de la mise en place d'une législation zoophile et de l'interdiction de ce spectacle le moyen de substituer aux comportements féroces de la plèbe, dignes des bull-dogs employés lors des matchs, le tempérament autrement plus docile et honnête des taureaux martyrs des combats ${ }^{49}$. La surveillance et le correctif apportés par ce biais aux mœurs du peuple doivent permettre l'avènement d'une nouvelle figure du pauvre, plus policée et mieux ajustée aux valeurs d'une bourgeoisie dont ces parlementaires minoritaires se pensent comme les représentants. Dans leur version de l'idée "animaliste» se découvre dès lors comme la projection impensée de principes de vision encore hétérodoxes. C'est l'affirmation implicite d'un modèle actualisé des rapports d'autorité qui ne peut alors se dire, au sein du champ du pouvoir, autrement que sous une forme euphémisée, telle qu’à travers cette question des cruautés infligées aux animaux. Bien que l'engagement au nom des bêtes dans le cénacle parlementaire ne se réduise pas à ces seules considérations, l'état des rapports de force propres à ce système de tensions influe de fait, pour une large part, sur la façon dont ces nouveaux porte-parole se saisissent de cette cause et la recentrent autour des loisirs et des pratiques populaires comme le bull-baiting.

La réception de cette forme actualisée de l'idéologie "animaliste» au sein de l'assemblée parlementaire s'avère des plus contrastée. Les différentes propositions zoophiles présentées à partir de 1800 sont systématiquement récusées jusqu'en 1822 par les membres des chambres législatives, l'indifférence amusée le disputant chez une majorité d'entre eux à l'agacement de voir mis en cause le sérieux de l'institution par l'introduction d'un sujet jugé trivial et indigne de leur attention. Au-delà de ces réactions, émerge dès les premiers débats un front d'opposition constitué d'une dizaine de parlementaires, ouvertement hostiles aux projets de lois et aux revendications portées par leurs promoteurs. Les critiques formulées insistent pour l'essentiel sur l'hypocrisie et l'absence de réciprocité des mesures soumises au parlement. Dans une période marquée par l'extension et le renouvèlement du black act et

49. Hansard, op. cit., London, T. C. Hansard, volume XXXVI, comprising the period from the twenty-ninth day of October, 1801, to the twelfth day of August, 1803, p. 845. 
des game laws, qui punissent impitoyablement la braconne perpétrée sur les terrains de chasse de la gentry et de l'aristocratie ${ }^{50}$, le projet de répression des jeux populaires comme le bull-baiting sous prétexte de leur cruauté a alors pour certains tous les traits de l'injustice. Ainsi l'explique William Windham à la chambre des communes à l'occasion de la discussion du Nationwide Ban on the Baiting of Bulls Bill, le 28 avril 1800. Récusant l'opinion de ses adversaires quant à l'exceptionnelle cruauté du jeu, il conteste les différences de traitement qu'introduiraient les projets de lois zoophiles entre les loisirs des grands et les amusements de la plèbe : «Les partisans de ce projet de loi ont proposé d'abolir le combat de taureaux en raison de sa cruauté. Il est assez curieux qu'un tel argument puisse être employé par un groupe de personnes ayant à leur disposition un code de lois des plus vexatoires pour la protection de leurs propres amusements. Je n'entends pas aujourd'hui condamner les lois sur la protection du gibier; mais quand des gentilshommes parlent de cruauté, je me dois de leur rappeler qu'elle appartient tout autant à la chasse qu'au combat de taureaux. [...] Les gens du peuple peuvent demander avec justice, pourquoi abolir le combat de taureaux, et protéger la chasse et le tir? Comment serons-nous perçus, si nous, qui avons à disposition toutes les formes d'amusement possibles, et qui pratiquons pourtant ces sports cruels, devenons les rigides censeurs des amusements des pauvres et les interdisons au prétexte de leur cruauté, alors qu'ils ne sont pas plus cruels que les nôtres? " ${ }^{51}$. Non pas que ces membres du parlement, pour la plupart plus proches politiquement du pôle conservateur que des jacobins, remettent ici en cause les privilèges de la noblesse ou les inégalités sociales constitutives de la société britannique. Au contraire, pour Windham, de même que pour d'autres défenseurs allégués d'un mode de vie traditionnel hérité des siècles passés, tels que George Canning ou William Cobbett, la condamnation morale et politique des combats de taureaux et de chiens menace l'assise même de l'organisation sociale comme des rapports d'autorité et justifie dès lors leur opposition ${ }^{52}$. Il faut se souvenir que dans les campagnes comme dans les villes, ces divertissements avaient constitué pendant longtemps autant d'événements mis en œuvre sous le patronage des puissants, tenus de fournir les animaux sacrifiés et les moyens matériels nécessaires à l'activité. Proches des cérémonies du Potlatch, transferts qui n'appellent pas à contrepartie mais suscitent la reconnaissance de leurs bénéficiaires en donnant à voir la générosité des "grands", ces rites étaient autant de moyens pour la gentry de mettre en scène sa puissance et son pouvoir auprès du peuple et de

50. Edward P. Thompson, Whigs and Hunters: The Origin of the Black Act, London, Penguin Books, 1975.

51. Hansard, op. cit., volume XXXV, p. 207.

52. Archibald Rosebery, The Windham Papers. The Life and Correspondence of William Windham, 1750-1810, London, H. Jenkins, 1913, volume II; William Cobbett, The Collected Social and Political Writings of William Cobbett, volume III. War, peace and the economy 1800-1805, London, Routledge/ Thoemmes Press, 1998. 
pérenniser sa domination ${ }^{53}$. En proposant de requalifier comme acte intolérable de cruauté le bull-baiting, dont la violence jusque-là était restée tolérée, voire même partiellement patronnée par l'élite patricienne, les soutiens au sein du parlement d'une idéologie "animaliste» actualisée tendent de fait, selon ces conservateurs, à bouleverser le fragile équilibre des relations entre les groupes sociaux.

L'opposition résolue de ces parlementaires au fil des années et des discussions successives des projets de lois zoophiles ne suffit pas toutefois à endiguer la dynamique progressive d'acceptation de l'idéologie "animaliste» remaniée au sein des chambres législatives. En témoigne d'ailleurs la redéfinition des objections formulées contre les propositions présentées dans les années 1820 : si les critiques dénoncent la tendance tyrannique de projets de lois cherchant à régenter la vie privée des citoyens, plus personne désormais ne questionne le stigmate de cruauté et de barbarie apposé à un jeu comme le bull-baiting ou ne remet en cause le caractère problématique des interactions de la plèbe avec les bêtes ${ }^{54}$. Persiste bien sûr pendant longtemps parmi les parlementaires et, plus largement, les membres de l'élite patricienne, une ambivalence certaine dans l'appréciation de ces formes brutales et sanglantes de sport. C'est sans doute le commentateur sportif Nimrod, chroniqueur et témoin fidèle de la vie aristocratique de l'époque, qui a le mieux restitué le mélange de fascination et de répulsion qu'éprouve alors l'élite sociale britannique à la vue de ces rituels de la plèbe impliquant des animaux : «Leur courage obstiné tout au long de la bataille, et leur détermination inébranlable à vaincre ou à mourir ont quelque chose de surnaturel... Il y a beaucoup à admirer dans ces combats, et plus encore dans le système dans son ensemble; mais je sens qu'il y a quelque chose d'à la fois répugnant et cruel dans ces affrontements interminables, même s'ils sont révélateurs du courage invincible de l'animal ${ }^{55}$. La fureur de la confrontation entre les bull-dogs dressés au combat et le puissant taureau trouve ainsi une résonance dans l'ethos guerrier d'une noblesse britannique qui, bien que pacifiée de longue date, conserve encore une attirance certaine pour les prouesses martiales ${ }^{56}$. Mais cette inclination se trouve contrebalancée de manière croissante par la pénétration des normes et des valeurs morales propres à la middle-class britannique, du fait entre autres de l'intensification des alliances, notamment matrimoniales, entre les représentants de la gentry et les familles de la grande bourgeoisie marchande et

53. Marcel Mauss, Essai sur le don. Forme et raison de l'échange dans les sociétés archaïques, Paris, PUF, 2007 (1 $1^{\text {re }}$ édition 1925); Edward P. Thompson, 'Patrician Society, Plebeian Culture', Journal of Social History, tome $7, \mathrm{n}^{\circ} 4,1976$, p. 382-405.

54. Cf. les débats autour des projets de lois présentés au début des années 1820 : Hansard's Parliamentary Debates, London, T. C. Hansard, 1829-1891, volume V (1821/04/03-1821/07/11); volume VII (1822/04/24-1822/08/06); volume IX (1823/05/01-1823/07/19).

55. Nimrod (Charles James Apperley), The Life of a Sportman, London, John Moore, 1948, p. 93, cité dans John Strachan, 'A New Style of Literature: Charles James Apperley ("Nimrod") and Late Georgian Sporting Biography', Romanticism, tome 19, n³ 3013, p. 310-326.

56. Roland Axtmann and Helmut Kuzmics, Autority, State, and National Character. The Civilizing Process in Austria and England, 1700-1900, Aldershot, Ashgate Publishing, 2007. 
financière. La généralisation de ces principes éthiques dans les rangs de l'élite patricienne, couplée à un processus plus avancé qu'ailleurs de pacification de l'aristocratie et de renforcement des formes d'autocontrôle des violences pulsionnelles, expliquent l'ambivalence des attitudes et les convictions partagées par un nombre de plus en plus important de représentants de ce groupe social du caractère problématique des relations des classes populaires avec les animaux. Se réalise ainsi dans les premières décennies du XIX ${ }^{\mathrm{e}}$ siècle la reconnaissance progressive parmi ces fractions de la classe dominante de la légitimité de l'idéologie, reconnaissance sans doute encore accentuée par les expériences pratiques de l'élite sociale. L'antinomie désormais ressentie par de nombreux parlementaires entre les jeux de la plèbe tels que le bull-baiting et leurs propres loisirs cynégétiques se nourrit alors des décalages effectifs suscités par l'évolution respective de ces activités. La tendance à l'esthétisation de la traque et à l'euphémisation de la violence qui transparait dans les récits de chasse au renard de l'époque suffit à se convaincre de l'ampleur des écarts entre les logiques d'exécution du combat de taureaux et l'expérience ressentie et vécue par les chasseurs à courre. Les longues pages consacrées à la description de la campagne anglaise et aux pérégrinations des hommes, chevaux et chiens à la poursuite de cet éclair de fourrure rouge jurant avec le vert des pâtures et bocages, y contrastent en effet avec les quelques lignes accordées à une mise à mort bien souvent évoquée de manière cursive et détournée ${ }^{57}$. L'acceptation par l'élite sociale britannique d'un porte-parolat des bêtes critiques des seules pratiques de la plèbe résulte donc aussi de l'horreur éprouvée vis-à-vis d'une violence progressivement oblitérée de leurs propres loisirs. La version de l'idéologie " animaliste» qui s'impose dans les premières décennies du XIX ${ }^{e}$ siècle a ainsi contribué à naturaliser l'arbitraire d'un processus de différenciation sociale des formes d'interaction avec l'animal, difficilement perceptible par les acteurs eux-mêmes.

La convergence des différents processus étudiés, depuis les bouleversements de la configuration du bull-baiting à un niveau microsocial jusqu'aux évolutions des rapports de force au sein du parlement et du champ du pouvoir, ont largement favorisé la légitimation d'une version de l'idée «animaliste» focalisée sur les pratiques des classes populaires et leurs interactions avec les animaux. Dans les premières décennies du XIX ${ }^{\mathrm{e}}$ siècle ces conceptions se diffusent ainsi en Grande-Bretagne, avec d'autant plus de succès qu'elles font échos aux grandes transformations structurelles qui affectent le Royaume-Uni. Le contexte est en effet marqué par l'effondrement progressif de la société agrarienne traditionnelle, sous les coups de boutoir du mouve-

57. Cf. Robert Smith Surtees, Hawbuck Grange, or the Sporting Adventures of Thomas Scott, esq., London, Bradbury, Agnew and co., 1847. 
ment des enclosures et du phénomène conjoint de concentration des terres entre les mains de grands propriétaires issus principalement de l'aristocratie et de la gentry. Face à ceux-ci, les petits paysans avaient su faire valoir jusquelà l'ancienneté de leur occupation des terres, et se prévalaient de tout un ensemble de rites, de traditions et de coutumes, parmi lesquels figurait en bonne place le bull-baiting ${ }^{58}$. L'idée de représentation des animaux se trouve alors mobilisée par l'élite patricienne dans ces conflits avec la "plèbe» rurale. Elle contribue à fragiliser encore un peu plus et à progressivement redéfinir ces rites et coutumes violents comme autant de pratiques illégitimes, s'articulant à tout un ensemble de traits - alcoolisme, impiété et non-respect du dimanche chômé, violences conjugales - constitutifs des figures en voie de formalisation du "mauvais pauvre» et des "classes dangereuses» ${ }^{59}$.

Dans ce moment de contestation des traditions culturelles des classes populaires, l'impossibilité d'adaptation matérielle et symbolique du bullbaiting à l'abaissement continué des seuils de tolérance à la violence et aux normes dominantes régissant le traitement et les interactions avec les bêtes précipite sa destruction. Malgré les actes de résistances des soutiens du sport - ainsi par exemple de la composition de nombreux chants locaux moquant les partisans de la zoophilie, ou de la poursuite des combats dans certains villages - son interdiction est finalement votée en $1835^{60}$, puis la pratique progressivement périclite et finit par disparaitre de la mémoire collective britannique. Aux alentours de 1850, avant même que de nouveaux loisirs populaires comme le music-hall ne viennent concurrencer les combats de chiens et de $\operatorname{coq} s^{61}$, elle n'est déjà plus pour de nombreux commentateurs qu'une vague et honteuse réminiscence de la barbarie de la "plèbe" britannique du tournant du siècle. La disparition de cette forme insulaire des combats d'animaux marque ainsi le triomphe et la naturalisation en Grande-Bretagne d'une acception de l'idéologie " animaliste» que le rituel violent, paradoxalement, a contribué à façonner.

Fabien Carrié est docteur en science politique et chercheur post-doctorant à l'Université Paris-Nanterre

58. Robert W. Macolmson, Popular Recreations in English Society. 1700-1850, Cambridge, Cambridge University Press, 1973.

59. Peter Gay, La culture de la haine, hypocrisie et fantasme de la bourgeoisie de Victoria à Freud, Paris, Plon, 1998.

60. Robert W. Macolmson, op. cit.

61. Fabrice Bensimon, «La culture populaire au Royaume-Uni, 1800-1914», Revue d'histoire moderne et contemporaine, 2001/5, $\mathrm{n}^{\circ}$ 48-4 bis, p. 75-91. 